引用格式: 翟志宏, 江民星, 常春英. 降水对蔬菜价格的冲击效应: 以广州为例 [J]. 资源科学, 2021, 43(2): 304-315. [Zhai Z H, Jiang M X, Chang C Y. Impact of precipitation on vegetable prices: Taking Guangzhou City as an example[J]. Resources Science, 2021, 43(2): 304-315.] DOI: 10.18402/resci.2021.02.09

\title{
降水对蔬菜价格的冲击效应 以广州为例
}

\begin{abstract}
翟志宏 ${ }^{1,2}$, 江民星 ${ }^{3,4,5}$, 常春英 ${ }^{6}$
(1. 暨南大学产业经济研究院, 广州 510632 ; 2 . 广东省气候中心, 广州 510080 ; 3 . 南京信息工程 大学商学院, 南京 210044 ; 4 . 南京信息工程大学江北新区发展研究院, 南京 210044 ; 5 . 南京信息 工程大学江苏人才强省建设研究基地, 南京 210044 ; 6. 广东省环境科学研究院, 广州 510045)
\end{abstract}

摘 要: 降水对蔬菜价格波动有重要影响, 分析降水的蔬菜价格冲击效应对菜价预测及市场供需调整具有重 要意义。本文首先构建了一个包含降水因素的蔬菜供需动态模型, 揭示逐日降水对菜价的影响机制, 并阐明了降 水对不同蔬菜冲击效应存在异质性的原因; 然后基于广州市2004-2018 年逐日的菜心、生菜和豆角 3 种蔬菜零售 价格数据及降水数据, 采用 VAR模型、脉冲响应函数和预测方差分解方法来验证上述影响机制, 并量化降水在月和 日尺度上对 3 种蔬菜价格的冲击效应。研究发现: 1) 一定条件下逐日过量降水对菜价具有正向冲击作用, 即导致菜 价上涨, 且冲击效应与蔬菜需求价格弹性呈反向关系。(2)菜价受降水的冲击在不同时间尺度上异质性明显, 日尺 度的冲击效应表现更为敏感, 降水对菜心、生菜和豆角价格冲击在日尺度上分别在第 $16 、 20$ 和 $10 \mathrm{~d}$ 达到最大, 随后 减弱; 而月尺度的冲击效应具有明显滞后性(滞后一个月), 冲击较强但持续性不强。(3) 日降水对菜心、生菜和豆角 价格波动的贡献率分别约 $8.3 \%$ 、 $18.4 \%$ 和 $1.0 \%$; 月 尺度降水相对日尺度降水而言, 对 3 种菜价的影响更大, 贡献率分 别约为 $24.0 \%$ 、 $18.1 \%$ 和 $10.7 \%$ 。最后, 本文提出了针对降水过量时期稳定蔬菜价格的政策建议。

关键词: 蔬菜价格; 冲击效应; 菜价波动; 降水; VAR 模型; 脉冲响应函数; 广州

DOI :10.18402/resci.2021.02.09

\section{1 引言}

民以食为天, 蔬菜价格变动关系到人民群众的 生活成本和福利水平, 是人们密切关注的重要民生 话题。研究表明蔬菜零售价格变化会导致农村和 城市居民消费福利的反方向变化 ${ }^{[1]}$, 而稳定的蔬菜 价格更利于提升农村居民的总福利 ${ }^{[2]}$ 。因此, 认识 和掌握不同蔬菜价格变化规律,有利于蔬菜价格监 测和预警, 实现蔬菜市场供需精准调控 ${ }^{[3,4]}$ 和蔬菜价 格稳定, 对保障民生利益具有重要意义。

影响蔬菜等农产品价格的因素大致可分为宏 观经济因素、市场因素及气象因素 3 类。宏观经济
因素主要包括农产品政策 ${ }^{[5,6]}$ 、居民收人 ${ }^{[7]}$ 、国际贸易 及其政策 ${ }^{[8-10]}$ 、农产品补贴 ${ }^{[11]}$ 、汇率与货币扩张 ${ }^{[12]}$ 等。 市场因素主要包括农产品供求关系、替代品价格和 竞争行为、交易形式和成本变化等。具体如下: 1 供求关系变化导致蔬菜价格波动。例如农产品供 求变化 ${ }^{[13,14]}$ 、蔬菜需求变动 ${ }^{[7]}$ 、受灾和环境风险 ${ }^{[15]}$ 、

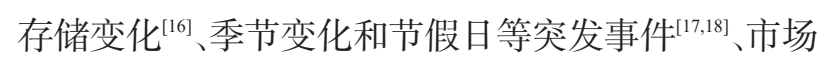
投机 ${ }^{[19]}$ 等因素引发供求变化,进而造成价格波动。 (2)替代品价格变化与竞争行为因素导致蔬菜价格 波动。许多研究表明,蔬菜价格的未来变化与前期 价格波动存在联系 ${ }^{[20]}$, 同时,受到替代品价格波动的

收稿日期: 2020-02-26 修订日期: 2020-07-05

基金项目: 国家自然科学基金项目(71903099);江苏省高等学校自然科学研究面上项目(19KJB610019);南京信息工程大学人才启动基金项 目(2018r089)。

作者简介: 翟志宏, 男, 江西九江人, 博士研究生, 高级工程师, 研究方向为气候经济学。E-mail: zhaizhgz@163.com 通讯作者: 江民星,男,江西九江人,博士,讲师,研究方向为产业经济学。E-mail: 18588847515@163.com 
影响 ${ }^{[21-25]}$ 以及农产品销售商销售竞争行为影响 ${ }^{[26]}$ 。 (3)交易形式和市场流通因素导致蔬菜价格波动。 如李崇光等 ${ }^{[2]}$ 分析 3 种交易模式下蔬菜价格形成机 制; 章胜勇等 ${ }^{[28]}$ 则发现加强中国南北蔬菜市场整合 可降低菜价波动, 缩短农产品供应链可有效降低新 鲜菜果的价格 ${ }^{[29,30]}$ 。(4)生产成本变化因素导致蔬菜 价格波动。例如, 城市道路桥梁公共设施密度和燃 料成本 ${ }^{[25]}$ 、流通成本对价格变化的影响 ${ }^{[3]}$ 。在气象 因素影响方面, 现有研究主要考察了降水、温度、日 照、风速和气候变化对蔬菜、水果等农产品价格影 响。蔬菜价格方面, 郭娜等 ${ }^{[32]}$ 发现黄瓜价格对降水 及温度变化的敏感程度较小, 对日照时数更为敏 感, 他们还发现蔬菜价格恢复力与蔬菜价格对上述 自然因素的敏感程度成反比 ${ }^{[33]}$; 薛正平等 ${ }^{[34]}$ 考察了 暴雨天数、暴雨降水量与台风乘积分别与青菜价格 涨跌之间的响应关系, 降水量、暴雨天数、平均最大 风速等天气要素变化与青菜价格涨幅均呈现显著 正相关 ${ }^{[35]}$; 而且, 在台风暴雨的天气中, 蔬菜价格通 常呈快速上涨、缓慢回落特征, 经 $20 \mathrm{~d}$ 左右恢复至 正常水平 ${ }^{[36]}$ 。水果价格方面, 袁斌等 ${ }^{\left[{ }^{37}\right.}$ 对比分析了 暴雨与温度对主产区与非主产区的鲜桃价格冲击 效应。其他农产品方面, Browna 等 ${ }^{[38]}$ 利用世界 51 个 发展中国家数据总结发现, 其中近 $20 \%$ 粮食短期价 格波动是显著受降水因素影响; 部分学者 ${ }^{[39-42]}$ 发现, 气候变化引起的极端天气, 如异常降水、干旱与温 度变化将冲击农产品价格。

降水是蔬菜价格不稳定的重要干扰源, 蔬菜种 植、生长、采收和流通等环节受降水影响明显, 且降 水较其他气象因素变化更具不确定性, 人工干预难 度大, 加剧了蔬菜价格的频繁波动。在理论研究方 面, 鲜有文献分析降水对蔬菜价格变动的作用机 制, 其内在影响机制尚不明晰; 实证研究方面, 虽然 部分文献 ${ }^{[32,37}$ 通过向量自回归模型(VAR 模型) 定量 考察了降水对蔬菜价格影响, 但未针对不同时间尺 度下的降水对蔬菜价格的冲击效应加以刻画, 也没 有揭示降水对不同蔬菜价格冲击异质性规律。另 外, 现有文献在研究农产品价格波动特征时, 多采 用年或月价格等长期数据 ${ }^{[43]}$ 。但事实上, 降水对长 期蔬菜价格影响机制与对日度短期价格影响机制 存在重要差别: 过量降水影响蔬菜生长、采收和运
输等环节使蔬菜上市量减少, 从而造成菜价短期波 动; 但在长期, 农户可调整蔬菜种植结构、种植周期 安排,并采取适应性生产措施从而可能影响蔬菜产 量及其价格。因此, 采用年度或月度蔬菜价格序列 并不能准确刻画降水的短期冲击效应, 需要用到日 度数据来更加准确地刻画这种短期冲击效应。

因此, 本文首先构建一个蔬菜供需动态模型, 通过该模型揭示逐日降水对菜价的影响机制, 分析 降水对不同蔬菜价格冲击效应异质性的原因; 其 次,利用2004-2018 年广州市菜心、生菜和豆角日 度零售价格及日度降水数据, 基于向量自回归模型 (VAR模型)、脉冲响应函数和预测方差分解方法验 证了上述影响机制, 并量化了降水对 3 种蔬菜在日 尺度上的短期冲击效应。作为对比研究, 本文构造 了月度数据, 进一步考察了降水对 3 种蔬菜在月尺 度的长期冲击效应。VAR 模型多用于分析时间序 列间的关系, 它能刻画当某一变量在 $t$ 期的扰动项 变动时, 对 $t$ 期以后各变量产生一连串的连锁作用, 它的分析结果有助于解释价格波动的原因和检验 波动是否符合经济理论 ${ }^{[20,32,34,37]}$ 。由于本文目的是考 察蔬菜价格在某期受降水冲击之后它将如何产生 变化, 故VAR模型适合本文研究。明确降水对不同 蔬菜冲击效应异质性原因及冲击效应, 可以为蔬菜 供应保障计划提供科学支撑, 有助于减轻市场调度 和流通压力, 保障蔬菜价格平稳、市场有序。

\section{2 理论模型}

参考 Gouel ${ }^{[10]}$ 、Fischer 等 ${ }^{[44]}$ 、Roberts 等 ${ }^{[45,46]}$ 学者 的研究, 本文假定蔬菜需求关于本期价格的线性函 数, 即蔬菜当日需求量决定于当日蔬菜价格, 需求 函数向右下倾斜: $D_{t}=c-d P_{t}$ 。 $D_{t}$ 与 $P_{t}$ 分别为蔬 菜市场需求量和价格, $c$ 和 $d$ 均为大于零的常数。 根据 Roberts 等研究 ${ }^{[45,46]}$, 本文认为供应商根据前 1 天价格决定当日蔬菜供应量, 并将降水因素纳人供 给函数当中,构造出如下形式的蔬菜供给函数:

$$
S_{t}= \begin{cases}a+\bar{h}\left(\bar{R}-R_{t}\right)+b P_{t-1}, & \text { 当 } \bar{R}<R_{t} ; \\ a+b P_{t-1}, & \text { 当 } \underline{R} \leqslant R_{t} \leqslant \bar{R} ; \\ a+\underline{h}\left(R_{t}-\underline{R}\right)+b P_{t-1}, & \text { 当 } \underline{R}>R_{t}\end{cases}
$$

式中: $S_{t}$ 为 $t$ 日市场蔬菜供给量; $P_{t-1}$ 为 $t-1$ 日蔬菜 价格; $a$ 和 $b$ 均为常数, 且 $b>0$, 表明当日蔬菜供给量 与前 1 天价格呈正方向变化; $R_{t}$ 为该区域 $t$ 日单位 
面积土地的降水量; $\bar{h}$ 和 $h$ 均为大于 0 常数, 表示单 位面积降水变化对蔬菜供给的影响系数,为分析简 便, 本文假定它们均等于 1 , 需强调本文基本结论并 不因它们的数值设定而发生改变。此外, 为了使市 场收玫均衡存在, 须有 $d>b$ 。蔬菜的种植、生长和采 收等过程一般存在一个适宜降水量区间 $[\underline{R}, \bar{R}]$ 。

考虑到降水过量比降水不足的人工干预更困 难, 前者对蔬菜供给影响更大, 因此以下将重点分 析过量降水冲击蔬菜价格的机制, 先分析初始降水 状态为最佳区间情形,再分析初始降水过量情形。 不妨令初始 $t$ 日处于适量降水区间, 降水量为 $R_{t}$, 则 均衡价格为: $P_{t}^{*}=(c-a) /(b+d)$ 。此后降水过量状 态持续 $k$ 天, 在 $k$ 天期间降水变化有 3 种 : 降水加强、 降水维持和降水减弱。

对于降水加强情形, 任意相邻两日蔬菜价格关 系为:

$$
\left.\overrightarrow{P_{t+i}}=\left(\sum_{m=1}^{i} \Delta_{t+m}+c-a-b \overrightarrow{P_{t+i-1}}\right)\right) / d, \quad i=1, \cdots, k
$$

式中: $\Delta_{t+m}=R_{t+m}-R_{t+m-1}>0$ 。由式(2)可得, 当满足 $\Delta_{t+i}+b\left(\overrightarrow{P_{t+i-1}}-\overrightarrow{P_{t+i-2}}\right)>0$ 条件时, 有 $\overrightarrow{P_{t+i}}>\overrightarrow{P_{t+i-1}}$, 因 此在一定条件下, 随着降水增强, 蔬菜价格逐日上 涨。一般地, 一个区域单位面积土地自然降水量和 土壤水分流失率大致相同, 可近似地认为该区域内 $\Delta_{t+1}$ 相等。因此, 蔬菜价格冲击效应差异实际上源 于不同蔬菜需求曲线斜率绝对值或需求价格弹性 的差异, 且弹性越大 (即 $d$ 越大), 降水过量对价格冲 击效应越小。

对于降水维持情形, 即 $\Delta_{t+i}=0(i=2, \cdots, k)$, 供 给线不发生移动, 根据市场出清条件, $P_{t+i+1}=$
$\left(c-a-\Delta_{t+1}-b P_{t+i}\right) / d$, 均衡条件下, $P_{t+i}=P_{t+i+1}$, 故 蔬菜价格将收玫至均衡, $P_{t+i}^{*}=\left(c-a+\Delta_{t+1}\right) /(b+d)$ 。

对于降水减弱情形,任意相邻两天蔬菜价格关 系如下：

$$
\overrightarrow{P_{t+i}}=\left(\Delta_{t+1}-\sum_{m=2}^{i} \Delta_{t+m}+c-a-b \overrightarrow{P_{t+i-1}}\right) / d, i=2, \cdots, k
$$

当满足 $-\Delta_{t+i}+b\left(\overrightarrow{P_{t+i-1}}-\overrightarrow{P_{t+i-2}}\right)<0$ 条件时, 据式 (3)有 $\overrightarrow{P_{t+i}}<\overrightarrow{P_{t+i-1}}$, 因此, 在一定条件下蔬菜价格随 着降水减弱将逐日下降。

若初始状态为降水过量情形,则初始供给线为 $S_{t}=a-\Delta_{t}+b P_{t}, \Delta_{t}=R_{t}-\bar{R}$, 初始均衡价格为 $P_{t}^{*}=\left(\Delta_{t}+c-a\right) /(b+d)$ 。该情形下实际是将初始降 水状态处于最佳区间平移 $\Delta_{t}$ 个单位, 之后重复上述 分析过程, 所得结论与上述所分析的初始降水状态 为最佳区间一致,故此处不再详述。

上述结论可以图形加以解释。图 $1 \mathrm{a} 、 1 \mathrm{~b} 、 1 \mathrm{c}$ 分 别表示降水加强、降水维持和降水减弱 3 种情形下 蔬菜价格波动情况, 初始降水状态均为最佳区间。 由于降水过量的初始状态与降水为最佳区间的初 始状态的分析相同,故此处不再重复分析。纵坐标 代表蔬菜需求量; 横坐标代表蔬菜价格; 图中阴影 部分表示实际降水量为最佳区间形成区域; $S_{t}$ 表示 该区域形成的最佳供给曲线,也是初始状态下供给 曲线; 阴影以下区域 (即供给线 $S_{t}$ 下方的区域) 表示 降水过量,该区域内的供给线表示降水过量后的供 给线; $D$ 为蔬菜市场需求曲线。

图 1a 显示降水逐期增强时菜价变动情况,最初 均衡点位于点 $A, t+1$ 日遭遇降水过量, 该日供给线 为 $S_{t+1}$ 。供应商按照上日价格向市场供应蔬菜数量

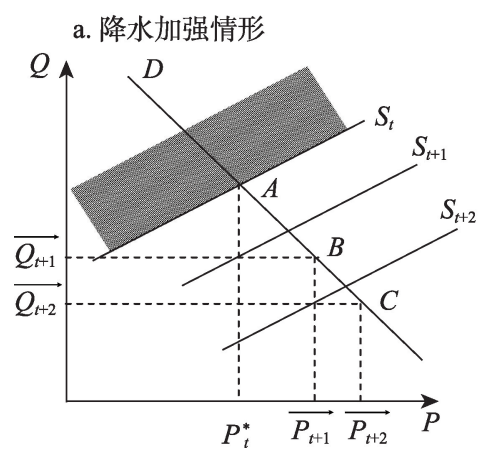

b. 降水维持情形

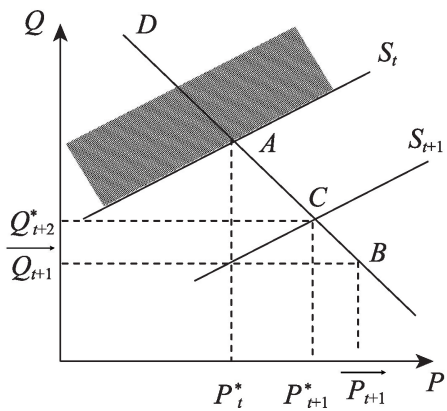

c. 降水减弱情形

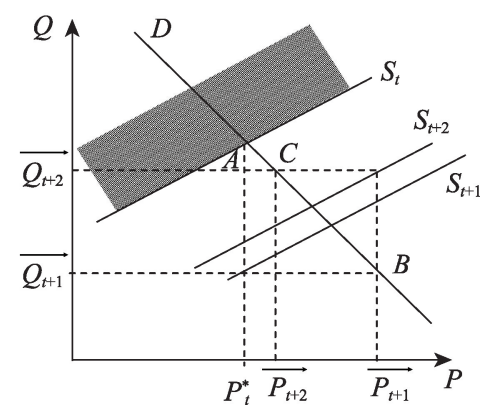

图 1 不同情形下降水对蔬菜价格冲击情况

Figure 1 Impact of precipitation on vegetable prices in different situations 
为 $\overrightarrow{Q_{t+1}}$, 它低于上日供给量, 结果以 $B$ 处的更高价格 $\overrightarrow{P_{t+1}}$ 实现市场出清; 随着降水进一步加强, $t+2$ 日供 给线继续下移至 $S_{t+2}$, 供应量低于上日, 将以 $C$ 处更 高的价格 $\overrightarrow{P_{t+2}}$ 实现新一轮市场出清。如果降水继 续加强, 供给线将继续下移, 重复上述过程。整个 过程可直观地发现, 其他条件不变的情况下, 降水 加强对每日蔬菜供应量具有负向冲击效应, 而对每 日蔬菜价格具有正向冲击效应。

图 $1 b$ 为降水维持情形下蔬菜价格变动情况。 降水过量导致实际供给线将下移至 $S_{t+1}, B$ 点代表 过量降水后市场出清, 出清价格和交易量分别为 $\overrightarrow{P_{t+1}}$ 和 $\overrightarrow{Q_{t+1}}$, 显然价格比初始状态更高, 而供应量比 初始状态更少。由于 $t+2$ 日降水维持 $t+1$ 日水平, 该日实际供给线不再移动, 并于 $C$ 处实现均衡, 均衡 价格为 $P_{t+2}^{*}$, 并且比上期价格要低。因此, 如果后 期降水继续保持不变, 且其他条件未发生变化, 蔬 菜供给线不会变动, 菜价将保持在均衡点 $C$ 的水平。

图 1c 代表降水减弱情形。 $S_{t+1}$ 表示 $t+1$ 日过 量降水后实际供给线,市场出清点为 $B$ 点。由于 $t+2$ 日降水减弱 (并非消失), 意味着最佳供给线下 移幅度比 $t+1$ 日下移幅度小, 因此 $S_{t+2}$ 必然处于 $S_{t}$ 与 $S_{t+1}$ 之间, 市场出清点从 $B$ 点变动到 $C$ 点, 价格从 $\overrightarrow{P_{t+1}}$ 下降为 $\overrightarrow{P_{t+2}}$, 更进一步地, 随着降水减弱, 价格 将继续下跌。

通过上述动态供需模型揭示了逐日降水对蔬 菜价格影响机制, 发现满足相应条件的情况下, 降 水对菜价具有正向冲击效应, 且冲击效应与蔬菜价 格需求弹性成反向关系, 以下将通过实证数据验证 上述结论,并进一步量化降水对菜价的冲击效应。

\section{3 模型设定与数据来源}

\section{1 模型设定}

脉冲响应函数 (Impulse Response Function, 简 称 IRF), 可以描述 VAR 系统对冲击 (新生) 扰动的动 态反应,并测算出变量间的滞后关系, 因而可以用 来分析降水对蔬菜价格持续性影响。预测误差的 方差分解 (Forecast-error Variance Decomposition, 简 称FEVD), 可将系统不同时期的预测误差方差分解 到各变量, 以了解各变量新冲击对模型内生变量的 相对重要性。因此, 本文主要采用VAR 模型和 IRF 方法开展实证分析, 利用 FEVD分析降水和菜价冲 击对菜价的贡献率。VAR模型数学形式如下:

$$
y_{t}=\boldsymbol{A}_{1} y_{t-1}+\cdots+\boldsymbol{A}_{p} y_{t-p}+\boldsymbol{B} x_{t}+\boldsymbol{\varepsilon}_{t}, t=1,2, \cdots, T(4)
$$
式中: $y_{t}$ 和 $x_{t}$ 分别是 $k$ 维内生变量 (菜价)和 $d$ 维外 生变量 (降水); $p$ 和 $T$ 分别是滞后阶数与样本个数; 矩阵 $\boldsymbol{A}_{j}(j=1,2, \cdots, p)$ 和矩阵 $\boldsymbol{B}$ 分别是 $k \times k$ 维和 $k \times d$ 维待估系数矩阵; $\boldsymbol{\varepsilon}_{t}$ 是 $k$ 维随机扰动列向量, 可 以同期相关, 但不与其滞后项相关, 也不与 $y_{t}$ 滞后 项相关。

此外, 为提高脉冲响应分析可靠性,在脉冲效 应分析之前采用格兰杰因果关系检验 (Granger causality test) 来检验降水与蔬菜价格是否存在统计意 义因果关系。格兰杰因果关系检验以序列有协整 关系为前提，常用的协整检验方法有基于残差的 E-G 两步法和基于系统的 Johansen 检验。由于此处 仅涉及单个协整关系的检验,因此采用基于残差的 E-G两步法。

\section{2 数据说明}

逐日降水资料为广州气象站 2004-2018 年的 时间序列数据, 来源于广东省气候中心。2004 2018 年广州本地菜心、生菜和豆角日度价格数据, 来源于广州市价格监测中心提供的广州市某蔬菜 市场逐日蔬菜零售监测数据。之所以选取广州上 述 3 种蔬菜, 主要有两点理由:一是广州每年蔬菜产 量、播种面积均为广东之首, 是广东及港澳地区蔬 菜的主要来源地,并且广州地处亚热带季风海洋性 气候区, 平均年降水量超过 $1800 \mathrm{~mm}$,年降水日数在 $150 \mathrm{~d}$ 左右, 可见广州蔬菜供应规模大且易受降水影 响;二是广东居民日常对上述 3 种蔬菜消费需求大， 几乎全年进行种植和交易, 菜心和生菜作为叶菜类 蔬菜代表,豆角作为瓜豆类蔬菜代表, 从而使本研 究具有代表性。

本文选取日度降水和日度菜价作为短期变量, 来研究降水的短期冲击效应; 同时作为对比研究, 本文选取月降水和月度菜价作为长期变量, 来刻画 降水的长期冲击效应。月度菜价通过日度菜价算 术平均得到。月度降水量 (rain_m) 和日度降水量 (rain_d)均采用原始数据进行分析。菜心 $(c x)$ 、生 菜 $(s c)$ 、豆角 $(d j)$ 等蔬菜价格由于不同年份间价格 波动较大, 需要先取自然对数, 使得数据更加平稳, 同时削弱了数据的异方差性,且不会改变价格数据 原有的变化特征。数据处理运用 STATA15.0 软件 完成。表 1 为变量的描述性统计结果。 


\section{表 1 变量描述性统计}

Table 1 Descriptive statistics of the variables

\begin{tabular}{clllcccc}
\hline \multicolumn{2}{c}{ 变量符号 } & 变量名称 & \multicolumn{1}{c}{ 变量取值 } & 均值 & 标准差 & 最小值 & 最大值 \\
\hline 月度 & rain_m & 降水量 & 月降水量 $/ \mathrm{mm}$ & 169.8 & 161.6 & 0.0 & 834.6 \\
& $\ln c x \_m$ & 菜心价格 & 月菜心价格, 取对数 & 1.675 & 0.376 & 0.800 & 2.867 \\
& $\ln s c \_m$ & 生菜价格 & 月生菜价格, 取对数 & 1.398 & 0.444 & 0.311 & 2.773 \\
& $\ln d j \_m$ & 豆角价格 & 月豆角价格, 取对数 & 1.908 & 0.356 & 1.059 & 2.989 \\
日度 & rain_d & 降水量 & 日降水量 $/ \mathrm{mm}$ & 5.6 & 15.2 & 0.0 & 222.1 \\
& $\ln c x \_d$ & 菜心价格 & 日菜心价格, 取对数 & 1.662 & 0.411 & 0.000 & 3.401 \\
& $\ln s c \_d$ & 生菜价格 & 日生菜价格, 取对数 & 1.383 & 0.477 & 0.000 & 3.584 \\
& $\ln d j \_d$ & 豆角价格 & 日豆角价格, 取对数 & 1.895 & 0.381 & 0.956 & 3.178 \\
\hline
\end{tabular}

\section{4 结果分析与讨论}

\section{1 基础检验}

4.1.1 平稳性与协整检验

基于VAR 模型分析降水和菜价的相关性之前, 需要对这两个时间序列分别进行平稳性检验。采 用 $\operatorname{ADF}$ (Augmented Dickey Fuller) 检验方法进行单 位根检验, 结果显示: 日度菜价、月度菜价和降水的 原始序列均在 $1 \%$ 显著性水平下通过平稳性检验。 在此基础上的 $\mathrm{E}-\mathrm{G}$ 两步法结果显示, 月度和日度降 水与 3 种蔬菜价格序列之间在 $1 \%$ 显著性水平下均 存在协整关系。

\subsection{2 滞后的阶数}

根据式 (4), VAR 模型需要确定内生变量的最 优滞后阶数。为获得更为稳健和可靠的结果, 综合 使用 LL、LR、FPE、AIC、HQIC 和 SBIC 等检验方法, 按照少数服从多数的原则, 选择最优阶数。因 FPE 与 AIC 对滞后阶数的估计可能偏高, HQIC 和 SBIC 给出的滞后阶数相对真实 ${ }^{[47]}$, 据此本文执行带*号最 多者为最优阶数的选择标准, 当*号一样多时以 HQIC 和 SBIC 对应的滞后阶数为准, 若估计的 VAR 模 型残差存在自相关则重新选择最优阶数。考虑到
数据序列样本数足够多, 本文将日度数据最大滞后 期设为 30 期, 月度数据最大滞后期设为 20 期。

表 2 报告了月度与日度的蔬菜价格最优的滞后 阶数, 月度菜心、生菜、豆角最优滞后期分别为 $12 、 8$ 和 12 期, 日度分别为 $8 、 12$ 和 19 期。在原序列平稳 的基础上, 检验结果显示, 所有特征根全部位于单 位圆之中, 故所建立的 VAR 模型稳定。此外, 估计 VAR 模型后还需要对其残差是否存在自相关进行 检验, 结果显示各序列白噪声检验 $1 、 2$ 阶在 5\%显著 性水平下拒绝自相关假设。在此基础上,进一步通 过格兰杰因果检验、脉冲响应函数、预测方差分解 等方法来分析降水对蔬菜价格序列之间的影响。

\subsection{3 格兰杰因果检验}

表 3 为 Granger 因果关系检验结果, 结果显示: 日度数据中降水是 3 种菜价变动的格兰杰原因 (单 向因果关系), 月度关系中降水对菜心表现为单向 因果, 而对生菜和豆角则为双向因果。从检验结果 来看, 在 $1 \%$ 显著水平下, 生菜与豆角月价格均与降 水互为格兰杰原因,但显然菜价变化是无法影响降 水的,这表明格兰杰检验难以识别生菜和豆角月度 价格与降水之间的长期因果关系。降水是菜心月

表 2 最优的滞后阶数

Table 2 Best lag order test results

\begin{tabular}{|c|c|c|c|c|c|c|c|c|}
\hline \multicolumn{2}{|c|}{ 变量 } & \multirow{2}{*}{$\frac{\mathrm{Lag}}{12}$} & \multirow{2}{*}{$\frac{\text { LL }}{-915.278}$} & \multirow{2}{*}{$\frac{\text { LR }}{19.903 *}$} & \multirow{2}{*}{$\frac{\text { FPE }}{646.366^{*}}$} & \multirow{2}{*}{$\frac{\mathrm{AIC}}{12.142^{*}}$} & \multirow{2}{*}{$\begin{array}{r}\text { HQIC } \\
12.534\end{array}$} & \multirow{2}{*}{$\frac{\text { SBIC }}{13.107}$} \\
\hline 月度 & $\ln c x \_m$ & & & & & & & \\
\hline & $\ln s c \_m$ & 8 & -971.266 & 10.183 & $1065.01 *$ & $12.645^{*}$ & 12.911 & 13.301 \\
\hline & $\ln d j \_m$ & 12 & -870.738 & $24.297^{*}$ & $369.118^{*}$ & $11.582 *$ & 11.974 & 12.547 \\
\hline \multirow[t]{3}{*}{ 日度 } & $\ln c x_{-} d$ & 8 & -16726.4 & 42.168 & $1.673^{*}$ & $6.190^{*}$ & $6.205^{*}$ & 6.232 \\
\hline & $\operatorname{lnsc\_ d}$ & 12 & -16859.5 & 8.862 & $2.046^{*}$ & $6.392 *$ & 6.414 & 6.454 \\
\hline & $\ln d j \_d$ & 19 & -14614.0 & 15.592 & $1.638^{*}$ & $6.169^{*}$ & 6.206 & 6.275 \\
\hline
\end{tabular}

注: *号代表相对应准则下的最优滞后阶数。 
表 3 Granger 因果关系检验结果

Table 3 Granger causality test results

\begin{tabular}{|c|c|c|c|c|}
\hline & 原假设 & $c h i^{2}$ & $d f$ & Prob $>c h i^{2}$ \\
\hline \multirow[t]{6}{*}{ 月度数据 } & rain_ $m$ 不是 $\ln c x \_m$ 的格兰杰原因 & 69.341 & 12 & 0.000 \\
\hline & lncx_m 不是 rain_m 的格兰杰原因 & 22.946 & 12 & 0.028 \\
\hline & 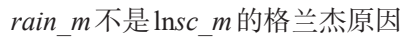 & 67.123 & 8 & 0.000 \\
\hline & lnsc_m 不是 rain_m 的格兰杰原因 & 21.523 & 8 & 0.006 \\
\hline & rain_m 不是 $\ln d j \_m$ 的格兰杰原因 & 49.436 & 12 & 0.000 \\
\hline & lndj_m 不是 rain_m 的格兰杰原因 & 39.801 & 12 & 0.000 \\
\hline \multirow[t]{6}{*}{ 日度数据 } & rain_d 不是 $\ln c x \_d$ 的格兰杰原因 & 71.249 & 8 & 0.000 \\
\hline & $\ln c x \_d$ 不是rain_d 的格兰杰原因 & 13.021 & 8 & 0.111 \\
\hline & rain_d 不是 $\ln s c \_d$ 的格兰杰原因 & 109.230 & 12 & 0.000 \\
\hline & lnsc_d不是rain_d 的格兰杰原因 & 13.865 & 12 & 0.309 \\
\hline & rain_d 不是 $\ln d j \_d$ 的格兰杰原因 & 52.739 & 19 & 0.000 \\
\hline & lndj_d 不是 rain_d 的格兰杰原因 & 34.912 & 19 & 0.014 \\
\hline
\end{tabular}

度价格变化的格兰杰原因, 反过来则不通过显著性 检验。因为从长期看, 农户应对气候变化的措施, 如根据雨水分布安排年内种植计划, 或者关闭低洼 易涝地区种植基地, 都会影响到当地蔬菜产量及价 格。而在日度数据序列中, 3 种蔬菜价格与降水的 格兰杰检验均认为降水是菜价变动的格兰杰原因
( $1 \%$ 显著水平下), 并且反过来菜价都不是降水变动 的格兰杰原因。这表明格兰杰检验较好地识别了 日度数据中蔬菜价格与降水之间的单向因果关系。

\section{2 降水对蔬菜价格波动的脉冲响应分析}

图 2 和图 3 分别展示了月度和日度尺度降水对 菜价的脉冲响应分析结果, 横轴为滞后期,纵轴表
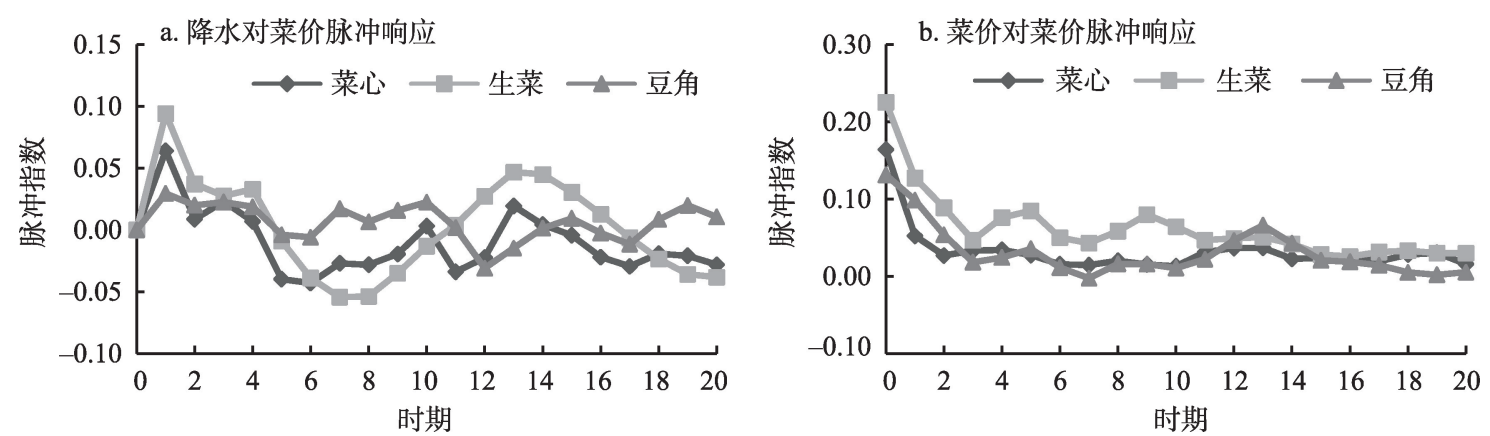

图 2 月度降水与菜价脉冲响应图

Figure 2 Impulse response function diagram of monthly precipitation and vegetable prices
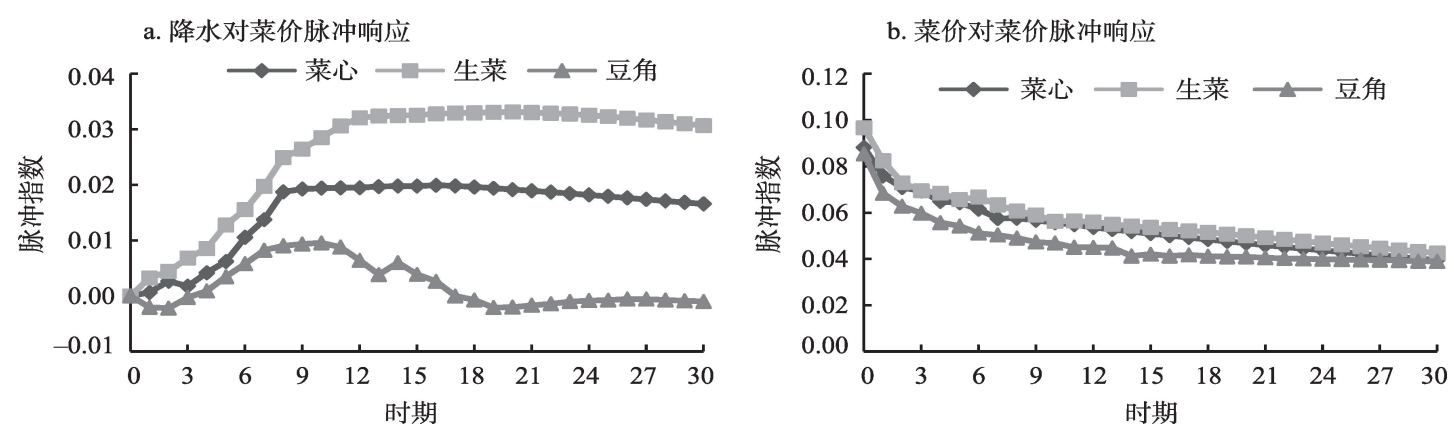

图 3 日度降水与菜价脉冲响应图

Figure 3 Impulse response function diagram of daily precipitation and vegetable prices 
示冲击程度(正交化的脉冲响应值)。

图 $2 \mathrm{a}$ 显示, 降水对当期蔬菜价格冲击效应为 0 , 在滞后 1 期出现正向冲击,滞后 2 4 期影响趋于消 失,表明当月降水对菜价的冲击效应滞后 1 期, 后续 影响减弱。在 4 期以后又出现波动, 原因可能是受 月降水变化影响而产生种植调整,如菜价提升刺激 菜农扩大种植面积,或灾害性降雨导致后续无法种 植。图 $2 b$ 显示, 3 种蔬菜价格波动对滞后 1 期的价 格存在较强正面冲击,滞后 2 期和 3 期迅速减弱, 从 第 4 期起, 影响维持在较低水平。刘凯 ${ }^{[48}$ 等利用 $\mathrm{ARCH}$ 类模型对北京市月度蔬菜价格进行分析, 发 现蔬菜价格受前期波动的正向影响,但持续时间较 短,这与本文的结果一致。

日度脉冲响应图则呈现出不同的规律。图 3a 显示,降水对当期蔬菜价格的影响为 0 , 从第 1 期开 始对菜心、生菜价格冲击持续为正且不断增加, 在 16 期和 20 期达到最大,之后缓慢减弱。本文结果与 文献[36,37]一致。豆角在前 2 期存在负响应,之后 变为正响应并持续增加,在第 10 期达到最大,之后 快速收敛,于第 17 期后基本消失。图 $3 \mathrm{~b}$ 显示, 3 种
蔬菜价格波动对滞后 1 期的价格存在较强的正面冲 击, 随后影响逐渐减弱, 但衰减速度慢于长期价格 的自身滞后惯性。图 3a 表明过量降水发生后, 3 种 蔬菜日度价格的脉冲指数刚开始均增加, 然后下降 并逐渐趋于平稳,这证实了理论模型中过量降水对 菜价具有正向冲击作用,并且随着降水逐渐减弱蔬 菜价格下降,并恢复至均衡水平的结论。一般而 言, 蔬菜替代品越少, 该蔬菜需求价格弹性越小。 菜心和生菜为广东地区非常受欢迎的两种蔬菜, 居 民需求量较大,并且可替代性弱于豆角,因此,它们 都比豆角的需求弹性更小; 另外,生菜储存难度大 于菜心, 需求更为刚性, 因而生菜弹性比菜心更 小。从图 $2 \mathrm{a}$ 和图 $3 \mathrm{a}$ 表明生菜脉冲指数(即降水对价 格冲击大小) 最大, 其次是菜心 豆角冲击效应最 小, 这与本文理论推导得到的降水对弹性较大的蔬 菜价格冲击效应较小结论契合。

\section{3 蔬菜价格预测方差分解}

月度数据的方差分解结果如表 4 所示, 结果显 示, 相对于蔬菜月度价格, 月度降水对菜价波动的 方差分解贡献较小, 月度降水对菜心价格冲击在第

表 4 月度数据方差分解结果

Table 4 Variance decomposition results of monthly data

\begin{tabular}{|c|c|c|c|c|c|c|}
\hline 时期 & $\begin{array}{c}\ln c x \_m \text { 对 } \ln c x \_m \\
\text { 的贡献 }\end{array}$ & $\begin{array}{c}\text { rain_m 对 } \ln c x \_m \\
\text { 的贡献 }\end{array}$ & $\begin{array}{c}\ln s c_{-} m \text { 对 } \ln s c_{-} m \\
\text { 的贡献 }\end{array}$ & $\begin{array}{c}\text { rain_m 对 } \ln s c \_m \\
\text { 的贡献 }\end{array}$ & $\begin{array}{c}\ln d j \_m \text { 对 } \ln d j \_m \\
\text { 的贡献 }\end{array}$ & $\begin{array}{c}\text { rain_m 对 } \ln d j \_m \\
\text { 的贡献 }\end{array}$ \\
\hline 1 & 1.000 & 0.000 & 1.000 & 0.000 & 1.000 & 0.000 \\
\hline 3 & 0.879 & 0.121 & 0.880 & 0.121 & 0.959 & 0.041 \\
\hline 4 & 0.870 & 0.130 & 0.875 & 0.125 & 0.945 & 0.056 \\
\hline 6 & 0.840 & 0.160 & 0.881 & 0.119 & 0.937 & 0.063 \\
\hline 7 & 0.804 & 0.196 & 0.871 & 0.129 & 0.937 & 0.063 \\
\hline 8 & 0.791 & 0.209 & 0.850 & 0.150 & 0.929 & 0.071 \\
\hline 9 & 0.779 & 0.221 & 0.833 & 0.167 & 0.928 & 0.072 \\
\hline 10 & 0.773 & 0.227 & 0.834 & 0.166 & 0.922 & 0.078 \\
\hline 12 & 0.760 & 0.240 & 0.841 & 0.160 & 0.911 & 0.089 \\
\hline 13 & 0.758 & 0.242 & 0.839 & 0.161 & 0.893 & 0.107 \\
\hline 14 & 0.759 & 0.241 & 0.829 & 0.172 & 0.900 & 0.101 \\
\hline 15 & 0.761 & 0.239 & 0.819 & 0.181 & 0.903 & 0.097 \\
\hline 16 & 0.764 & 0.236 & 0.815 & 0.185 & 0.903 & 0.097 \\
\hline 17 & 0.759 & 0.241 & 0.815 & 0.185 & 0.903 & 0.097 \\
\hline 18 & 0.749 & 0.251 & 0.816 & 0.184 & 0.901 & 0.099 \\
\hline 19 & 0.747 & 0.253 & 0.814 & 0.186 & 0.900 & 0.100 \\
\hline 20 & 0.746 & 0.254 & 0.808 & 0.192 & 0.892 & 0.108 \\
\hline
\end{tabular}


显示, 相对于蔬菜日度价格, 日度降水对菜价波动 的方差分解贡献仍然较小, 到第 30 期时降水对生菜 价格和菜心价格方差分解贡献度约 $18.4 \%$ 和 $8.3 \%$, 而对豆角价格在第 11 期达到 1\%此后基本稳定,因 而再次验证了降水对弹性较大的蔬菜价格冲击效 应较小的结论。日度价格波动对自身波动的变化 贡献度更大,第 2 期 3 种蔬菜价格自身波动贡献度 均在 $99.9 \%$ 以上, 之后缓慢下降,到第 30 期菜价自 身波动贡献也均在 $80 \%$ 以上,仍起主要作用。文献 [37]也有类似发现,在主产区,气温和降水对鲜桃日 价格贡献率在 $10 \%$ 和 $1 \%$ 以上,价格自身变化的贡献

表 5 日度数据方差分解表

Table 5 Variance decomposition results of daily data

\begin{tabular}{|c|c|c|c|c|c|c|}
\hline 时期 & $\begin{array}{c}\ln c x_{-} d \text { 对 } \ln c x_{-} d \\
\text { 的贡献 }\end{array}$ & $\begin{array}{c}\text { rain_d 对 } \ln c x \_d \\
\text { 的贡献 }\end{array}$ & $\begin{array}{c}\operatorname{lnsc\_ d} d \text { 对 } \ln s c_{-} d \\
\text { 的贡献 }\end{array}$ & $\begin{array}{c}\text { rain_d 对 } \operatorname{lnsc\_ d} \\
\text { 的贡献 }\end{array}$ & $\begin{array}{c}\ln d j \_d \text { 对 } \ln d j \_d \\
\text { 的贡献 }\end{array}$ & $\begin{array}{c}\text { rain_d 对 } \ln d j \_d \\
\text { 的贡献 }\end{array}$ \\
\hline 1 & 1.000 & 0.000 & 1.000 & 0.000 & 1.000 & 0.000 \\
\hline 2 & 1.000 & 0.000 & 0.999 & 0.001 & 1.000 & 0.000 \\
\hline 3 & 1.000 & 0.000 & 0.999 & 0.001 & 0.999 & 0.001 \\
\hline 4 & 1.000 & 0.000 & 0.997 & 0.003 & 1.000 & 0.001 \\
\hline 5 & 0.999 & 0.001 & 0.995 & 0.005 & 1.000 & 0.000 \\
\hline 6 & 0.998 & 0.002 & 0.991 & 0.009 & 0.999 & 0.001 \\
\hline 7 & 0.995 & 0.005 & 0.986 & 0.014 & 0.998 & 0.002 \\
\hline 8 & 0.991 & 0.009 & 0.979 & 0.021 & 0.996 & 0.004 \\
\hline 9 & 0.983 & 0.017 & 0.968 & 0.032 & 0.994 & 0.006 \\
\hline 10 & 0.977 & 0.023 & 0.957 & 0.043 & 0.992 & 0.008 \\
\hline 11 & 0.971 & 0.029 & 0.946 & 0.054 & 0.990 & 0.010 \\
\hline 12 & 0.965 & 0.035 & 0.935 & 0.066 & 0.989 & 0.012 \\
\hline 13 & 0.961 & 0.039 & 0.923 & 0.077 & 0.988 & 0.012 \\
\hline 14 & 0.956 & 0.044 & 0.912 & 0.088 & 0.988 & 0.012 \\
\hline 15 & 0.952 & 0.048 & 0.903 & 0.097 & 0.988 & 0.012 \\
\hline 16 & 0.948 & 0.052 & 0.894 & 0.106 & 0.988 & 0.012 \\
\hline 17 & 0.945 & 0.055 & 0.886 & 0.114 & 0.988 & 0.012 \\
\hline 18 & 0.942 & 0.059 & 0.878 & 0.122 & 0.989 & 0.011 \\
\hline 19 & 0.938 & 0.062 & 0.871 & 0.129 & 0.989 & 0.011 \\
\hline 20 & 0.936 & 0.064 & 0.864 & 0.136 & 0.989 & 0.011 \\
\hline 21 & 0.933 & 0.067 & 0.858 & 0.142 & 0.990 & 0.010 \\
\hline 22 & 0.931 & 0.069 & 0.852 & 0.148 & 0.990 & 0.010 \\
\hline 23 & 0.929 & 0.072 & 0.847 & 0.154 & 0.990 & 0.010 \\
\hline 24 & 0.927 & 0.074 & 0.841 & 0.159 & 0.990 & 0.010 \\
\hline 25 & 0.925 & 0.075 & 0.836 & 0.164 & 0.991 & 0.009 \\
\hline 26 & 0.923 & 0.077 & 0.832 & 0.168 & 0.991 & 0.009 \\
\hline 27 & 0.921 & 0.079 & 0.827 & 0.173 & 0.991 & 0.009 \\
\hline 28 & 0.920 & 0.080 & 0.823 & 0.177 & 0.991 & 0.009 \\
\hline 29 & 0.918 & 0.082 & 0.819 & 0.181 & 0.991 & 0.009 \\
\hline 30 & 0.917 & 0.083 & 0.816 & 0.184 & 0.992 & 0.008 \\
\hline
\end{tabular}


率则达到 $60 \%$ 以上。

此外, 本文还进行了两种稳健性检验: 第一种 是样本分割法, 即将本文原始样本均匀分割进行稳 健性检验; 第二种则是替换变量法, 即采用同一市 场另 3 种相似蔬菜 (白菜、油麦菜和茄子) 价格数据 进行稳健性检验, 发现结果依然稳健 ${ }^{\mathbb{D}}$ 。

\section{5 结论与政策建议}

\section{1 结论}

本文主要考察了降水对蔬菜价格影响机制和 冲击效应, 首先构建一个蔬菜动态供需模型, 分析 了逐日降水对菜价影响机制, 并揭示了不同类型蔬 菜冲击效应的异质性原因; 然后基于广州市菜心、 生菜和豆角的零售价格数据及降水数据, 通过 VAR 模型、脉冲响应函数和预测方差分解方法, 考察了 降水对菜价波动在日和月尺度上的时间变化特征， 证实了降水对上述菜价影响的作用机制, 并量化两 个时间尺度下的降水对蔬菜价格的冲击效应。本 文主要结论有:

(1) 在一定条件下, 逐日过量降水对菜价具有 正向冲击作用, 冲击效应大小与蔬菜需求价格弹性 呈反向关系。对于具有区域性偏好的蔬菜, 当地居 民消费频率高而替代品种少, 需求弹性较小, 从而 降水对其价格短期冲击效应较大。因此稳定蔬菜 价格的重点是保障当地偏好较强的蔬菜供应, 以防 当地主要蔬菜品种价格在短期内剧烈波动。

(2) 日尺度降水对菜心、生菜和豆角价格冲击 分别在第 16,20 和 $10 \mathrm{~d}$ 达到最大, 随后减弱; 月尺度 的降水对菜价的冲击效应具有明显滞后性(滞后一 个月), 冲击较强, 但持续性不强。因此, 蔬菜价格 受降水的冲击在不同时间尺度上存在显著差异, 即 在较小的时间尺度上更为敏感, 较大的时间尺度上 则相对滞后。此外, 对于叶菜类等易腐蔬菜而言, 无论在日尺度还是月尺度上, 其受降水冲击的持续 时间均长于瓜豆类等耐储蔬菜。

（3）日尺度降水对菜心、生菜、豆角价格波动的 贡献分别约 $8.3 \% 、 18.4 \%$ 和 $1.0 \%$, 月尺度降水的贡 献分别约为 $24.0 \% 、 18.1 \%$ 和 $10.7 \%$ 。虽然蔬菜价格 受降水冲击在较小的时间尺度上表现更为敏感, 但 冲击幅度较小, 由于长期的积累效果使得蔬菜价格 的波动在较大时间尺度上受降水因素影响更大。

\section{2 政策建议}

决策者可利用上述降水对菜价影响规律, 动态 监测蔬菜供应链中的生产、采购、运输、销售等环 节, 提前制定应对方案保障蔬菜稳定供应, 通过实 施有差别供应保障计划, 利于减轻市场调度和流通 压力, 保障蔬菜价格平稳有序, 可以采取的措施 包括:

(1)结合气象预报制定供应方案。暴雨来临 前, 加大菜篮子的外采、调运、收储, 做好叶菜类、茄 果类、瓜菜类等极端天气下价格变动较大的蔬菜供 给保障, 同时利用好根茎类等蔬菜易周转、不易腐 烂等特性确保供应。

(2)优先支持叶菜类设施种植。针对受冲击大 的蔬菜品种发展设施种植,增强其防灾能力, 保障 不利天气下的蔬菜供应, 减缓蔬菜价格受气象灾害 的冲击, 平抑菜价的剧烈波动, 以保护农民以及消 费者的切身利益。

(3)建立差异化的蔬菜供应保障计划。时间周 期上考虑长短结合, 先根据月度冲击效应制定月度 菜价稳定目标, 进而确定保障供应总量目标, 然后 根据日度菜价冲击情况定量投放, 避免蔬菜保障供 应短缺或过剩。品类上确定不同蔬菜供应保障的 优先级别。首先对于叶菜类蔬菜, 其耐储性更弱, 其受降水冲击更敏感,因而供应保障计划启动时间 应为优先级别; 其次对于耐储蔬菜且对降水敏感度 更小的菜种, 如豆角、茄子、黄瓜等茄果类和瓜菜 类, 及土豆、山药和红萝卜等根茎类蔬菜,受降水的 冲击效应较小,供应保障响应时间可迟于叶菜类蔬 菜; 最后居民蔬菜偏好很大程度上决定了蔬菜需求 弹性大小,故需要按照当地居民偏好制定供应保障 优先级计划。

\section{参考文献(References):}

[1] 罗超平, 牛可, 但斌. 蔬菜价格波动的农村与城镇居民福利效 应: 基于省际面板数据模型[J]. 农村经济, 2017, (4): 100-107. [Luo C P, Niu K, Dan B. Welfare effects of rural and urban residents on vegetable price fluctuations: Based on provincial pane data model[J]. Rural Economy, 2017, (4): 100-107.]

[2] 罗超平, 牛可, 李凌杰, 等. 蔬菜价格波动对农村居民福利效应 研究: 基于省际面板数据模型分析[J]. 价格理论与实践, 2017, (3): 66-69. [Luo C P, Niu K, Li L J, et al. Effects of vegetable

(1) 因篇幅限制未展示稳健性检验过程与结果, 如有需要, 请向作者索取 
price fluctuation on rural residents' welfare: Based on provincial panel data model[J]. Price: Theory \& Practice, 2017, (3): 66-69.]

[3] 李优柱, 李崇光, 李谷成. 中国蔬菜价格预警系统研究[J]. 农业 技术经济, 2014, (7): 79-88. [Li Y Z, Li C G, Li G C. Research on vegetable price warning system in China[J]. Journal of Agrotechnical Economics, 2014, (7): 79-88.]

[4] 牟子平, 吴文良, 雷红梅. 寿光农业结构类型与资源可持续利用 的技术对策 [J]. 资源科学, 2004, 24(6): 152-157. [Mou Z P, Wu W L, Lei H M. Types of agricultural structure and technical countermeasure for the sustainable utilization of resources in Shouguang County[J]. Resources Science, 2004, 24(6): 152-157.]

[5]张永强, 彭有幸, 周宁. 预期相对净收益视角下的蔬菜播种面积 变化分析: 基于2011-2017年蔬菜主产省区面板数据[J]. 农业经 济与管理, 2020, (1): 25-33. [Zhang Y Q, Peng Y X, Zhou N. Analysis on changes of vegetable planting areas from perspective of expected relative net income: Based on analysis of panel data of main vegetable production provinces and regions from 2011 to 2017[J]. Agricultural Economics and Management, 2020, (1): 25-33.]

[6] Enciso S R A, Fellmann T, Dominguez I P, et al. Abolishing biofuel policies: Possible impacts on agricultural price levels, price variability and global food security[J]. Food Policy, 2016, 61: 926.

[7] 韩旭东, 王若男, 崔梦怡, 等. 中国规模农户设施农业经营状况 及影响因素 [J]. 资源科学, 2020, 42(4): 749-762. [Han X D, Wang R N, Cui M Y, et al. An empirical analysis on the development status and influencing factors of facility agriculture of largescale farmers[J]. Resources Science, 2020, 42(4): 749-762.]

[8] 彭佳颖, 谢锐, 赖明勇. 国际粮食价格对中国粮食价格的非对称 性影响研究[J]. 资源科学, 2016, 38(5): 847-857. [Peng J Y, Xie R, Lai M Y. A study on the asymmetric impacts of international grain prices on domestic grain prices in China[J]. Resources Science, 2016, 38(5): 847-857.]

[9] Anderson K, Nelgen S. Trade barrier volatility and agricultural price stabilization[J]. World Development, 2012, 40(1): 36-48.

[10] Gouel C. Trade policy coordination and food price volatility[J]. American Journal of Agricultural Economics, 2016, 98(4): 10181037.

[11] 罗超平, 李伟毅, 翟琼. 外部冲击对蔬菜价格波动的影响: 基于 面板向量自回归模型(PVAR)的实证分析[J]. 中国蔬菜, 2013, (10): 16-22. [Luo C P, Li W Y, Zhai Q. Impact of external shock on fluctuation of vegetable price: Based on inter-provincial panel data VAR model[J]. China Vegetables, 2013, (10): 16-22.]

[12] 岳瑞雪, 谭砚文. 蔬菜价格波动的共同影响因子与宏观决定因 素[J]. 华南农业大学学报(社会科学版), 2019, 18(3): 79-91. [Yue R X, Tan Y W. Common factors and determinants of vegetable price fluctuation[J]. Journal of South China Agricultural University (Social Sciences Edition), 2019, 18(3): 79-91.]

[13] Karali B, Irwin S, Isengildina-Massa O. Supply fundamentals and grain futures price movements[J]. American Journal of Agricultur- al Economics, 2020, 102(2): 548-568.

[14] 包玉泽, 于顷, 周怡, 等. 中国蔬菜产业的布局及其演化研究: 1990-2014年[J]. 干旱区资源与环境, 2018, 32(11): 53-58. [Bao Y Z, Yu Y, Zhou Y, et al. Layout and evolutionary of China's vegetable industry: from 1990 to 2014[J]. Journal of Arid Land Resources and Environment, 2018, 32(11): 53-58.]

[15] Asche F, Oglend A, Selland K. Price dynamics in biological production processes exposed to environmental shocks[J]. American Journal of Agricultural Economics, 2017, 99(5): 1246-1264.

[16] Asche F, Oglend A, Zhang D J. Hoarding the herd: The convenience of productive stocks[J]. Journal of Futures Markets, 2015, 35(7): 679-694.

[17] 赵安平, 王大山, 肖金科, 等. 蔬菜价格时间序列的分解与分析: 基于北京市 2002-2012 年数据[J]. 华中农业大学学报(社会科 学版), 2014, (1): 49-53. [Zhao A P, Wang D S, Xiao J K, et al. Time series decomposition and analysis of vegetable prices: Based on data of Beijing from 2002 to 2012[J]. Journal of Huazhong Agricultural University (Social Sciences Edition), 2014, (1): 49-53.]

[18] 宋长鸣, 李崇光, 许军. 法定节假日会加剧蔬菜价格波动吗?[J]. 农业技术经济, 2017, (7): 116-121. [Song C M, Li C G, Xu J. Will legal holidays exacerbate vegetable price fluctuation?[J]. Journal of Agrotechnical Economics, 2017, (7): 116-121.]

[19] 王双进. 近期蔬菜价格波动特征、主要影响及成因分析[J]. 价格 理论与实践, 2016, (4): 102-105. [Wang S J. Analysis on recent vegetable prices fluctuation characteristics, main influence and reason[J]. Price: Theory \& Practice, 2016, (4): 102-105.]

[20] 罗超平, 王钊, 翟琼. 蔬菜价格波动及其内生因素: 基于 PVAR 模型的实证分析[J]. 农业技术经济, 2013, (2): 22-30. [Luo C P, Wang Z, Zhai Q. Vegetable price fluctuations and their endogenous factors: Empirical analysis based on PVAR model[J]. Journal of Agrotechnical Economics, 2013, (2): 22-30.]

[21] 赵翠萍. 中国城乡蔬菜价格联动机制实证分析[J]. 农业技术经 济, 2012, (6): 80-86. [Zhao C P. An empirical analysis of the linkage mechanism of urban and rural vegetable prices in China[J]. Journal of Agrotechnical Economics, 2012, (6): 80-86.]

[22] 王钊, 姜松. 中国蔬菜价格变动的空间计量分析[J]. 农业技术经 济, 2013, (11): 4-14. [Wang Z, Jiang S. Spatial econometric analysis of vegetable price changes in China[J]. Journal of Agrotechnical Economics, 2013, (11): 4-14.]

[23] 辛士波, 王笛. 耐储蔬菜与反季蔬菜价格波动的联动效应分析: 以北京市为例[J]. 北京工商大学学报(社会科学版), 2018, 33 (1): 52-60. [Xin S B, Wang D. Linkage effect of price fluctuation in shelf-stable vegetables and anti-season vegetables: Taking Beijing City as example[J]. Journal of Beijing Technology and Business University (Social Sciences), 2018, 33(1): 52-60.]

[24] 宋长鸣, 李崇光. 农产品市场的整合与替代研究: 以蔬菜为例 [J]. 中国农村经济, 2012, (11): 78-87. [Song C M, Li C G. Research on the integration and substitution of agricultural products market: Take vegetables for example[J]. Chinese Rural Economy, 
2012,(11): 78-87.]

[25] Shively G, Thapa G. Markets, transportation infrastructure, and food prices in Nepal[J]. American Journal of Agricultural Economics, 2017, 99(3): 660-682.

[26] 张否, 王娜, 吴金超. 中国蔬菜批发行业结构, 行为及绩效研究: 以山东寿光到北京的蔬菜流通为例[J]. 农业经济问题, 2018, (2): 115-126. [Zhang L, Wang N, Wu J C. Study on the structure, behavior and performance of vegetable wholesale industry in China: Taking vegetables circulation from Shouguang Shandong to Beijing as an example[J]. Issues in Agricultural Economy, 2018, (2): 115-126.]

[27] 李崇光, 肖小勇, 张有望. 蔬菜流通不同模式及其价格形成的比 较: 山东寿光至北京的蔬菜流通跟踪考察[J]. 中国农村经济, 2015, (8): 53-66. [Li C G, Xiao X Y, Zhang Y W. Comparison of different models of vegetable circulation and their price formation: Follow- up inspection of vegetable circulation in Shandong Shouguang to Beijing[J]. Chinese Rural Economy, 2015, (8): 53-66.]

[28] 章胜勇, 何怡蒙, 肖小勇. 交易成本视角下中国南北蔬菜市场整 合研究[J]. 农业技术经济, 2019, (8): 79-88. [Zhang S Y, He Y M, Xiao X Y. The study of north and south spatial market integration of vegetable in China from the perspective of transaction cost [J]. Journal of Agrotechnical Economics. 2019, (8): 79-88.]

[29] Aysoy C, Kirli D H, Tumen S. How does a shorter supply chain affect pricing of fresh food? Evidence from a natural experiment[J]. Food Policy, 2015, 57: 104-113.

[30] 六靖, 陈犧. 中国蔬菜价格波动的特征、原因及调控思路研究 [J]. 宏观经济研究, 2020, (4): 142-152. [Bian J, Chen X. A study on the characteristics, reasons and regulation of vegetable price fluctuation in China[J]. Macroeconomics, 2020, (4): 142-152.]

[31] 纪龙, 李崇光, 章胜勇. 中国蔬菜生产的空间分布及其对价格波 动的影响 [J]. 经济地理, 2016, 36(1): 148-155. [Ji L, Li C G, Zhang S Y. The spatial distribution of vegetable production in China and its impact on vegetable price volatility[J]. Economic Geography, 2016, 36(1): 148-155.]

[32] 郭娜, 问英杰. 天气因素对蔬菜价格恢复力的影响: 以石家庄市 本地黄瓜为例 [J]. 中国生态农业学报, 2015, 23(6): 785-792. [Guo N, Yan Y J. Impact of weather on vegetable price resilience: A case study of the local cucumber in Shijiazhuang City[J]. Chinese Journal of Eco-Agriculture, 2015, 23(6): 785-792.]

[33] 郭娜, 刘芳芳, 闰英杰. 蔬菜价格恢复力的供给因素影响分析 [J]. 价格理论与实践, 2016, (3): 60-63. [Guo N, Liu F F, Yan Y $\mathrm{J}$. Analysis on the impact of supply factors on vegetable price resilience[J]. Price: Theory \& Practice, 2016, (3): 60-63.]

[34] 薛正平, 李军, 孙燕, 等. 气象条件对青菜价格的影响及其关系 模型[J]. 上海农业学报, 2014, 30(5): 114-119. [Xue Z P, Li J, Sun Y, et al. Influence of meteorological conditions on the prices of Chinese cabbage and their relational model[J]. Acta Agriculturae Shanghai, 2014, 30(5): 114-119.]

[35] 薛正平, 李军, 张皓, 等. 上海青菜价格与梅雨关系初析 [J]. 上海
农业学报, 2009, 25(2): 86-88. [Xue Z P, Li J, Zhang H, et al. Preliminary analysis on the relationship between the Chinese cabbage price in Shanghai and the meteorological factors of rainy season[J]. Acta Agriculturae Shanghai, 2009, 25(2): 86-88.]

[36] 薛正平. 台风暴雨和暖冬对绿叶菜价格影响初探[J]. 大气科学 研究与应用, 2008, (1): 44-51. [Xue Z P. Impact of typhoon-rainstorm and warm-winter on the green leaf vegetable price[J]. Atmospheric Science Research and Application, 2008, (1): 44-51.]

[37] 袁斌, 陈超. 天气变化视角下易腐农产品价格波动研究[J]. 干旱 区资源与环境. 2020, 34(5): 147-153. [Yuan B, Chen C. Price fluctuation of perishable agricultural products from the perspective of weather change[J]. Journal of Arid Land Resources and Environment, 2020, 34(5): 147-153.]

[38] Brown M E, Kshirsagar V. Weather and international price shocks on food prices in the developing world[J]. Global Environmental Change, 2015, 35: 31-40.

[39] Bradbear C, Friel S. Integrating climate change, food prices and population health[J]. Food Policy, 2013, 43: 56-66.

[40] Willenbockel D. Extreme weather events and crop price spikes in a changing climate: Illustrative global simulation scenarios[J]. Oxfam Policy and Practice: Climate Change and Resilience, 2012, 8 (2): $15-74$

[41] Götz L, Djuric I, Nivievskyi O. Regional price effects of extreme weather events and wheat export controls in Russia and Ukraine [J]. Journal of Agricultural Economics, 2016, 67(3): 741-763.

[42] Tack J, Holt M T. The influence of weather extremes on the spatial correlation of corn yields[J]. Climatic Change, 2016, 134(1): 299309.

[43] 许世卫, 李哲敏, 李干琼, 等. 农产品市场价格短期预测研究进 展[J]. 中国农业科学, 2011, 44(17): 3666-3675. [Xu S W, Li Z M, Li G Q, et al. Advances in research of short-term forecasting methods of agricultural product price[J]. Scientia Agricultura Sinica, 2011, 44(17): 3666-3675.]

[44] Fischer C, Guttormsen A G, Smith M D. Disease risk and market structure in salmon aquaculture[J]. Water Economics and Policy, 2017, DOI: 10.1142/S2382624X16500156.

[45] Roberts M J, Schlenker W. World supply and demand of food commodity calories[J]. American Journal of Agricultural Economics, 2009, 91(5): 1235-1242.

[46] Roberts M J, Schlenker W. Identifying supply and demand elasticities of agricultural commodities: Implications for the US ethanol mandate[J]. American Economic Review, 2013, 103(6): 22652295.

[47] Lutkepohl H. New Introduction to Multiple Time Series Analysis [M]. Berlin: Springer, 2005.

[48] 刘凯, 穆月英, 韩婷. 蔬菜价格波动及风险研究: 以北京为例 $[\mathrm{J}]$. 中国蔬菜, 2018, (2): 63-70. [Liu K, Mu Y Y, Han T. Vegetables prices fluctuation and risk study: A case study in Beijing[J]. China Vegetables, 2018, (2): 63-70.] 


\title{
Impact of precipitation on vegetable prices:
}

\section{Taking Guangzhou City as an example}

\author{
ZHAI Zhihong ${ }^{1,2}$, JIANG Minxing ${ }^{3,4,5}$, CHANG Chunying ${ }^{6}$ \\ (1. Institute of Industrial Economics, Jinan University, Guangzhou 510632, China; 2. Guangdong Climate Center, Guangzhou \\ 510080, China; 3. Business School, Nanjing University of Information Science \& Technology, Nanjing 210044, China; \\ 4. Development Institute of Jiangbei New Area, Nanjing University of Information Science \& Technology, Nanjing 210044, \\ China; 5. Research Center for Prospering Jiangsu Province with Talents, Nanjing University of Information Science \& \\ Technology, Nanjing 210044, China; 6. Guangdong Provincial Academy of Environmental Science, Guangzhou 510045, China)
}

\begin{abstract}
Precipitation variations have important impacts on vegetable prices. Identifying and analyzing the shock effects of precipitation on vegetable prices are of great significance for vegetable prices forecasting and market supply and demand adjustment. Based on a dynamic model of vegetable supply and demand with precipitation factors, we revealed the mechanism of daily precipitation impulse on the price of vegetables and the cause of the heterogeneity of precipitation impact on different vegetables. Subsequently, based on daily and monthly retail vegetable prices (cabbage, lettuce, and asparagus bean) and precipitation data in Guangzhou City from 2004 to 2018, we used vector autoregression (VAR), impulse response function (IRF), and forecast error variance decompositions to verify the identified mechanism, and analyzed the effect of precipitation on the prices of the three vegetables. The results are as follows. (1) Under certain conditions, daily excess precipitation has a positive impact, which will stimulate vegetable prices, and the impact is inversely related to the price elasticity of vegetable demand. (2) The impact of precipitation on the price of vegetables has clear heterogeneity on different temporal scales, and the daily scale is more sensitive than the monthly scale. Daily precipitation impacts on cabbage, lettuce, and asparagus bean prices reach their maximum at 16, 20, and 10 day, respectively and then weakened. Monthly precipitation impact, which is strong, is reflected in the next month but not lasting. (3) Compared to daily precipitation, monthly precipitation has a greater impact on vegetable prices. Daily precipitation impacts on cabbage, lettuce, and asparagus bean contribute $8.3 \%, 18.4 \%$, and $1.0 \%$ of the price fluctuations, respectively, while monthly precipitation impacts contribute to a higher degree of $24.0 \%, 18.1 \%$, and $10.7 \%$. According to the results, this article proposed serval countermeasures to ensure the stable supply of vegetables.
\end{abstract}

Key words: vegetable price; shock effect; vegetable price fluctuation; precipitation; vector autoregression (VAR); impulse response function (IRF); Guangzhou 